\title{
The effect of hand posture on swimming efficiency
}

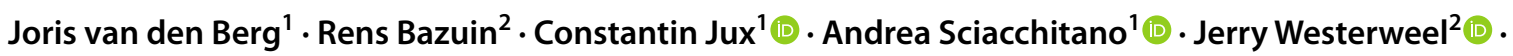 \\ Willem van de Water ${ }^{2}$ (1)
}

Received: 16 June 2021 / Revised: 11 October 2021 / Accepted: 14 October 2021 / Published online: 16 November 2021

(c) The Author(s) 2021

\begin{abstract}
Our quest is for the thumb and finger positions that maximize drag in front crawl swimming and thus maximize propulsion efficiency. We focus on drag in a stationary flow. Swimming is in water, but using Reynolds similarity the drag experiments are done in a wind tunnel. We measure the forces on real-life models of a forearm with hands, flexing the thumb and fingers in various positions. We study the influence on drag of cupping the hand and flexing the thumb. We find that cupping the hand is detrimental for drag. Swimming is most efficient with a flat hand. Flexing the thumb has a small effect on the drag, such that the drag is largest for the opened (abducted) thumb. Flow structures around the hand are visualized using robotic volumetric particle image velocimetry. From the time-averaged velocity fields we reconstruct the pressure distribution on the hand. These pressures are compared to the result of a direct measurement. The reached accuracy of $\approx 10 \%$ does not yet suffice to reproduce the small drag differences between the hand postures.
\end{abstract}

\section{Graphical Abstract}
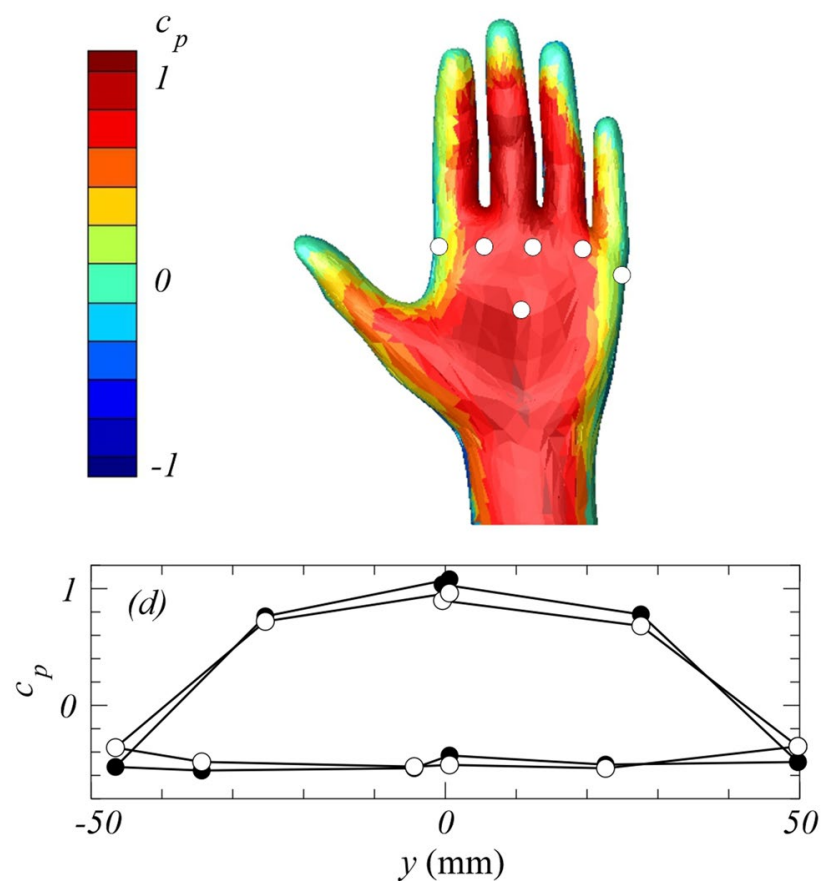

Willem van de Water

w.vandewater@tudelft.nl

Extended author information available on the last page of the article 


\section{Introduction}

Humans are not created for swimming. Their hands and feet lack fins, which are a tremendous aid in propulsion. However, they can change their hand posture: spreading fingers, extending (abducting) the thumb and cupping the hand. All with the purpose of increasing drag. In front crawl swimming increasing drag increases thrust and gives the same thrust at lower relative velocities, both resulting in enhanced swimming efficiency.

The effect of hand posture on static drag is expected to be small. Still, even a slight improvement of propulsion efficiency is significant for (top) swimmers. The hand with attached forearm is a complex object, and quantifying and understanding drag around such an object is a challenge for fluid dynamics. Of direct relevance are accurate drag measurements in a stationary flow. Insight in flow structures is provided by three-dimensional particle image velocimetry (PIV), and, as will be illustrated in this paper, it even allows for a faithful reconstruction of the pressure distribution on the hand.

Many studies on swimming hydrodynamics were concerned with the effect of spreading the fingers and flexing the thumb. Most experiments (Schleihauf 1979; Sidelnik and Young 2006) and numerical simulations (Minetti et al. 2009; Marinho et al. 2010; Lorente et al. 2012; Bilinauskaite et al. 2013; Vilas-Boas et al. 2015) concerned stationary drag in a steady flow. Instead, unsteady motion involving an accelerating robotic arm was explored by Sidelnik and Young (2006). The consensus now is that a small finger spread leads to enhanced drag (Sidelnik and Young 2006; Minetti et al. 2009; Marinho et al. 2010; Lorente et al. 2012; van Houwelingen et al. 2017). Variation of the thumb position has been tried by Takagi et al. (2001) who finds a larger drag of the hand with closed (adducted) thumb than for the hand with opened (abducted) thumb. Conversely, the opposite tendency was found by Marinho et al. (2009) using computational fluid dynamics. These partly conflicting results inspired the experiments of this paper.

In view of the superior drag of hemispheres with the concave opening facing the oncoming flow, it might be expected that cupping the hand helps enhance drag. To the best of our knowledge, there are no studies on the influence of hand cupping.

The drag on an object is expressed by the drag coefficient $C_{\mathrm{D}}, C_{\mathrm{D}}=F_{\mathrm{D}} / \frac{1}{2} \rho V^{2} A$, with $F_{\mathrm{D}}$ the drag force, $V$ the flow velocity, $\rho$ the fluid mass density, and $A$ the area of the object projected onto the main flow direction. The dependence of $C_{\mathrm{D}}$ of a spherical cap on its depth is documented in the textbook by Hoerner (1965). The drag has a maximum for a cup which is a perfect hemisphere $(h / d=1 / 2$, where $h$ and $d$ are defined in Fig. 1). At maximum the $C_{\mathrm{D}}$ is $20 \%$
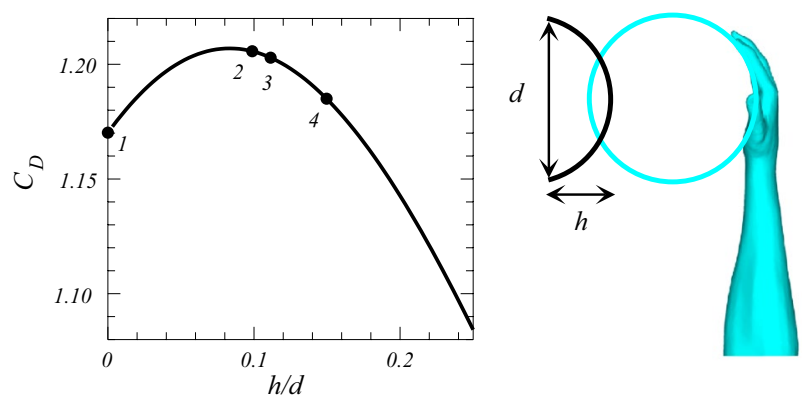

Fig. 1 The drag of a cupped disk as a function of its depth. The data are from the textbook by Hoerner (1965), but with a crucial twist. The drag coefficient is now plotted, normalized on the true area, and not the projected area as in Hoerner (1965). The cupped hands are fitted to a sphere, which defines the radius of curvature. The equivalent value of $h / d$ is determined by the radius of curvature and the length of the hand. The numbered dots correspond to the hands that were actually studied in the experiment (see Fig. 3).

larger than that of a circular disk with the same projected area. The question is whether this carries over to hand and finger curvature.

A caveat is that the $C_{\mathrm{D}}$ of a cup is defined with respect to its projected area, whereas in cupping hands, the surface area remains the same, while the radius of curvature changes. The variation of $C_{\mathrm{D}}$ with curvature, but now with respect to the surface area, is shown in Fig. 1. At its maximum it is now 3\% higher than that of a flat disk. Still, a 3\% increase in drag would result in a worthwhile improvement of swimming efficiency.

Determining the effects of finger and thumb position on swimming efficiency is challenging because the effects are so small. For experiments, wind tunnels are preferred. While forces and force moments can be measured accurately, the Reynolds numbers match those of real life. An enormous advantage is the absence of free surface effects which complicate experiments in water. At the small velocities in this experiment, the flow of air is incompressible.

For our experiment we scanned many hand-arm configurations, greatly benefitting from the public domain project "Make Human" (MakeHuman-Team 2001-2016), which provides a virtual human model with natural joints. We have used its forearm, flexed its thumb and fingers in the desired configuration, and made 3D prints. Two of those prints were equipped with 16 pressure holes, providing information about the pressure distribution on the hand and fingers.

We will first measure drag on models with cupped hands and hands with open and closed thumb. Next, we will map out the 3D mean flow around the hand and forearm using robotic volumetric PIV (Jux et al. 2018, 2020) and use it to verify a model for the flow between the fingers and to reconstruct the pressure on the hand. 
The effect of finger spreading has been understood by analyzing the flow between the fingers as it is driven by the pressure drop over the hand, a description reminiscent of the Betz theory of the actuator disk (Betz 1966; Westerweel et al. 2016; van Houwelingen et al. 2017). Much as the Betz theory predicts an optimum wind rotor efficiency as a function of the transparency of the actuator disk, does this hand model predict an optimum drag as a function of the finger spreading. So far, the effect of finger spreading has been studied through its influence on (static) drag. These measurements will not be repeated here. However, a map of the flow field allows verification of a key ingredient of the Betz model.

We have organized this paper as follows: in Sect. 2 we describe the manufacturing and shaping of the hand models. The wind tunnel drag balance setup is described in Sect. 3 . In Sect. 3.1 we study the mean drag as a function of the hand curvature, while scanning the angle of attack. Similarly, in Sect. 3.2 we measure the drag and lift as a function of the thumb abduction. Detailed structure of the full threedimensional mean flow using tomographic particle image velocimetry is discussed in Sect. 4 . This even allows reconstruction of the time-averaged pressure distribution on the hand, which is presented in Sect. 5.

\section{Hand models}

The full-scale 3D-printed arm models share the same forearm, while the finger and thumb positions are varied. All cupped hands have a $5^{\circ}$ finger spreading, as this was found optimal (van Houwelingen et al. 2017). The models are created with the open-source 3D computer program MakeHuman that is generally used for the prototyping of human models (MakeHuman-Team 2001-2016). The program uses a detailed human skeleton including its bones and joints. The cupped shapes are created by flexing the end points of the phalanges (digital bones in the hand) onto known curved shapes. Summarizing, the following hand models were studied; the numbers correspond to those of Figs. 3 and 4:

1. A flat hand. A plane perpendicular to the hand palm intersects the end point of the middle finger's phalanx that is nearest to the hand palm. All end points of the other phalanges are pushed onto this same plane.

2. Neutral hand. The phalanges are in a neutral position as generated by MakeHuman; this can be considered a hand at rest.

3. Curved hand, radius of curvature $R=0.12 \mathrm{~m}$. The end points of the phalanges lie on a sphere with the diameter of a basketball (diameter $D=0.24 \mathrm{~m}$ ). The sphere is placed at the hand palm side, with the end point of the middle finger's phalanx that is nearest to the hand palm fixed on it. From here, the positions of all other phalanges are chosen.

4. Curved hand, $R=0.095 \mathrm{~m}$. Equal to the basketball model, but now the used sphere has the smaller diameter of a handball $(D=0.19 \mathrm{~m})$.

For these hands the thumb is fixed in its neutral (abducted) position (see the front view in Fig. 6). In addition, two models were constructed with the fingers in the neutral position, one with adducted (closed) thumb, and one with the thumb abducted. For all hands, the drag coefficient is always with respect to the projected area $\left(A=0.04170 \mathrm{~m}^{2}\right)$ of the forearm with the neutral hand.

The six arm models are created by a 3D printer with a printing resolution of $120 \mu \mathrm{m}$ (Shapeways 2018), which we take as a roughness height. Surface roughness influences the critical Reynolds number $\operatorname{Re}_{\mathrm{cr}}$ where flow separation occurs. The roughness height to diameter ratio is $\approx 12 \times 10^{-3}$ for the hand or forearm and $\approx 6 \times 10^{-3}$ for a finger. Compared to a smooth cylinder with $\mathrm{Re}_{\mathrm{cr}} \lesssim 2.8 \times 10^{5}$, the work of Nakamura and Tomonari (1982) implies a value of $\mathrm{Re}_{\mathrm{cr}}$ which is a factor $\approx 2$ to $\approx 2.5$ smaller. To reduce reflections, the surface of the two models studied for the effect of the thumb position was painted black, which improved the surface smoothness.

\section{Drag measurements}

The Reynolds numbers relevant for swimming can be realized readily in wind tunnels since a flow velocity of $V=1 \mathrm{~m} / \mathrm{s}$ in water corresponds to $V=15 \mathrm{~m} / \mathrm{s}$ in air. Experiments were done in the low turbulence tunnel (LTT) at the Delft University of Technology, a facility used for accurate measurements of drag and lift in air flows with a very low free stream turbulence $(0.015 \%)$ ) (Timmer and van Rooij 2003). The test section cross section in these experiments is $1.8 \times 1.0 \mathrm{~m}^{2}$. The used arm-hand models have a negligible model blockage ratio of $1.9 \%$. The length of the test section is $2.6 \mathrm{~m}$. Along the walls of the test section, a laminar boundary layer develops, which is $\approx 1.2 \mathrm{~cm}$ thick at $x=2.5 \mathrm{~m}$ from its starting point. The arm-hand models are mounted with the forearm base flush on the force sensor plate, which itself is also flush with the upper wall of the test section (see Fig. 2). Due to the absence of a strut, the model not only experiences drag and lift forces, but also a force component in the $z$-direction (normal to the tunnel wall).

Yaw angles could be set with a precision of $0.5^{\circ}$, while forces and force moments can be determined with an accuracy of $0.01 \mathrm{~N}$ and $0.01 \mathrm{Nm}$, respectively. This results in a relative accuracy of $0.2 \%$ for the measured drag on our hand models at a free stream velocity of $15 \mathrm{~m} / \mathrm{s}$. The zero of the yaw angle $\varphi=0$ is defined such that the hand palm is 

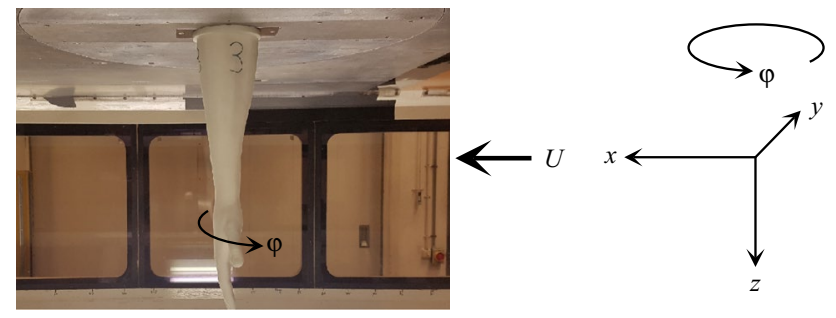

Fig. 2 A hand model in the wind tunnel test section and the used coordinate system. The yaw angle $\varphi$ is positive for the thumb leading into the main flow direction, while $\varphi=0$ for the hand palm perpendicular to the main flow direction. The model is mounted flush with the force sensor plate, which itself is flush with the upper wall of the test section
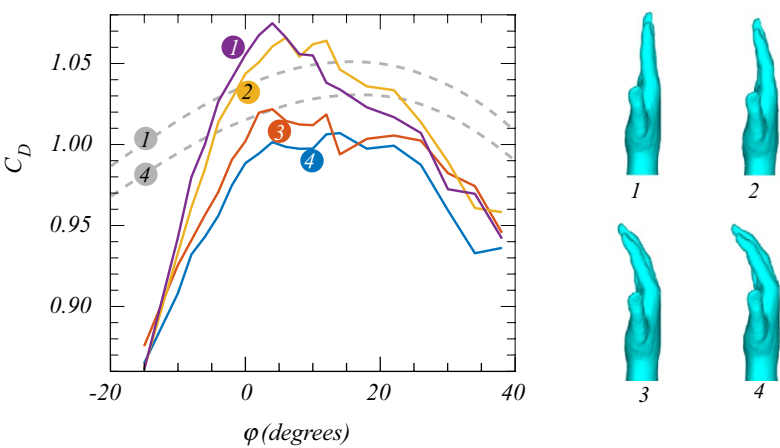

Fig. 3 (Color online) The drag coefficients of a forearm with cupped hands as a function of the yaw angle $\varphi$. The projected area of the flat hand 1 is larger than that of the strongest curved hand 4 . The dashed lines indicate the variation of this projected area with yaw angle. They have been normalized to $C_{\mathrm{D}}=1.05$ at maximum projected area of the flat hand. The typical reproducibility of the drag curves is shown in Fig. 4b

perpendicular to the mean flow (points in the $-x$ direction); with increasing $\varphi$ the thumb rotates into the incoming main flow.

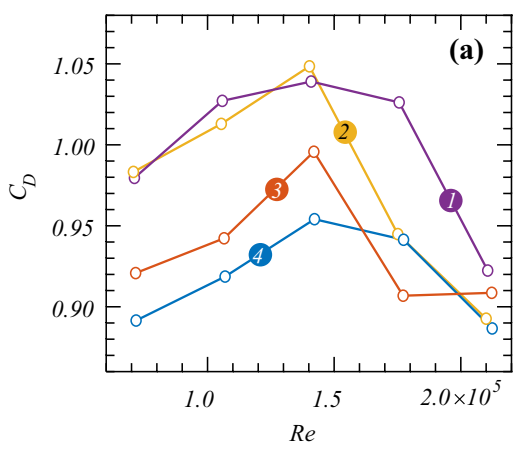

Fig. 4 (Color online) a The maximum drag coefficients of a forearm with cupped hands as a function of the Reynolds number. The numbers refer to a flat hand (1), a neutral hand (2), a hand curved around a basketball (3) and a hand curved around a handball (4) (see Fig. 3).
A typical experiment consists of a scan of yaw angles, starting at $\varphi=-20^{\circ}$ and increasing to $\varphi=60^{\circ}$. At each new angle, the flow was allowed to settle for $10 \mathrm{~s}$, after which data were registered for $20 \mathrm{~s}$ at a rate of $5 \mathrm{~Hz}$; these readings were averaged. In the experiments of Sec. 3.2, angular scans were done both for increasing and decreasing $\varphi$. The Reynolds numbers, based on the width $0.1 \mathrm{~m}$ of the hand, are in the range $\operatorname{Re}=[0.7-2.1] \times 10^{5}$. Therefore, flow separation around the hand and forearm is expected. The angle $\varphi$ where separation occurs may differ in a scan with increasing $\varphi$ from that in a scan with decreasing $\varphi$, such that force-angle curves may show hysteresis.

\subsection{Drag of cupped hands}

The influence of hand curvature on the drag coefficient at a main flow velocity of $15 \mathrm{~m} / \mathrm{s}\left(\operatorname{Re}=1.1 \times 10^{5}\right.$, which is typical in human swimming) is shown in Fig. 3 for yaw angles ranging from $-20^{\circ}$ to $40^{\circ}$. A striking observation is that the effect of curvature is a reduction of the drag, contrary to the suggestion of Fig. 1. Curvature will reduce drag by almost $6 \%$, and swimming with a flat hand is slightly more efficient than swimming with a neutral hand.

The drag coefficient was always computed using the projected area of the neutral hand. However, the projected area of a hand depends on its curvature. It is largest for the flat hand and smallest for the strongest curved hand 4 . The smaller measured drag of more strongly curved hands could then be a trivial geometric effect. Therefore, the projected area of these two extreme cases is also plotted in Fig. 3, demonstrating that the change in drag is not a simple geometric effect and has a true hydrodynamical origin.

In contrast to Schleihauf (1979) and Takagi et al. (2001), maximum drag does not occur at $\varphi=0^{\circ}$, where the hand palm is perpendicular to the flow, but instead at values $\varphi \approx 5 \ldots 10^{\circ}$ where the thumb is leading. The drag in Fig. 3 has a large contribution of the forearm whose cross section

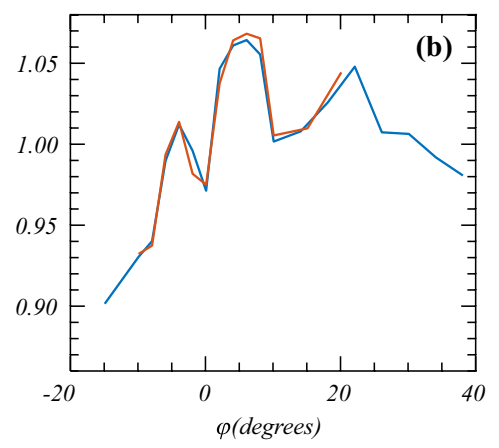

b Drag coefficient of a neutral (2) hand as a function of yaw angle $\varphi$ at a main air velocity of $20 \mathrm{~m} / \mathrm{s}\left(\operatorname{Re}=1.4 \times 10^{5}\right)$. The two curves are repetitions of the same angular scan. Large jumps in $C_{\mathrm{D}}$ are most probably due to flow separation 
Fig. 5 (Color online) Wake behind the hand with abducted (a) and closed (b) thumb in a horizontal plane at $z=116 \mathrm{~mm}$ below the tip of the middle finger

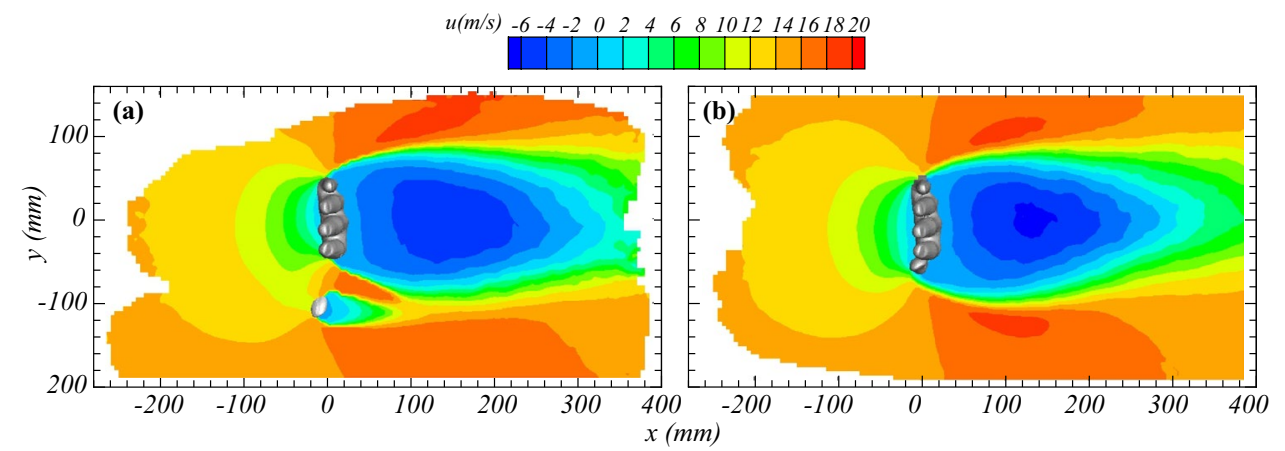

resembles a tilted ellipse. Consequently, the total drag has a maximum when the normal on the hand makes a small angle with the flow, while the lift vanishes at $\phi \approx 12^{\circ}$. However, what matters is the change of the drag (and lift) with different hand postures. We have found no significant variation with hand cupping of the lift force. The same conclusion about the detrimental effect of hand cupping on drag holds for the dimensionless force moment $C_{M_{y}}$, which emphasizes the force on the hand.

The ordering of drag forces on the hand models remains at other Reynolds numbers, as is illustrated in Fig. 4a; however, at the largest Re the results are less accurate due to larger (turbulent) force fluctuations.

The Reynolds numbers of the flow around the forearm are in the interval where the boundary layer turns turbulent, and drag crises may be observed. This is illustrated in Fig. 4b where $C_{\mathrm{D}}$ suddenly changes at particular values of $\varphi$. These phenomena are reproducible for the same hand model and repeated experiments, but slightly change for the models with a smoother skin which will be discussed in Sec. 3.2.

In conclusion, in spite of the expectation based on the enhanced drag of hemispherical objects, no such effect was found for cupped hands. On the contrary, swimming with flat hands is most efficient. Of course, a caveat is that this conclusion is reached in wind tunnel experiments which measure static drag, whereas real-life swimming is dynamic.

\subsection{Drag as a function of thumb abduction}

In the context of hand posture, a second question is about spreading (abduction) of the thumb. The idea is that flexing the thumb away from the hand changes the drag of the hand and thus influences swimming efficiency. It was studied experimentally by Takagi et al. (2001) and in computer simulations by Marinho et al. (2009), who conclude that thumb abduction decreases drag by $9 \%$ and $5 \%$, respectively. Both studies were on isolated hands. In the wind tunnel experiments of Takagi et al. (2001) force data were obtained from the measured pressure distribution over the hand. Whereas the effect of thumb position on $C_{\mathrm{D}}$ was found to be small, a more significant effect on lift was found in Schleihauf (1979), Takagi et al. (2001). For yaw angles $\varphi=40^{\circ}$, Takagi et al. (2001) found a $70 \%$ increase in $C_{\mathrm{L}}$ for the adducted thumb, while a $100 \%$ decrease was found for $\varphi=-60^{\circ}$ with the little finger leading. These partly conflicting results deserve further experiments.

The wakes behind the hand with abducted and closed thumb are shown in Fig. 5. The way in which they are measured will be discussed in Sect. 4. When closing the thumb, the oncoming flow, which previously passed in between thumb and hand palm, is now forced around the thumb. This results in an increased size of the stagnating region on the hand palm, close to the thumb. The circulating wake is observed for both cases, but it appears stronger in case of the closed thumb, as judged by the higher values of reversed flow at thumb height. The question now is how these qualitative observations relate to the change of drag and lift with thumb abduction.

The results of the force measurements are shown in Fig. 6. We have scanned the yaw angle for increasing and decreasing $\varphi$, which provides an idea about the reproducibility of the experiments. The drag of the arm and hand with abducted thumb is slightly ( $2 \%$ ) but significantly larger than that of the closed thumb, contrary to what was found by others (Takagi et al. 2001; Marinho et al. 2009). Since our $C_{\mathrm{D}}$ values are for the arm and hand together, the relative effect for the hand alone would be $\approx 4 \%$. The relative difference in $C_{\mathrm{D}}$ for the two thumb positions is larger than the relative accuracy of the wind tunnel force balance. The projected area of the model with abducted thumb is also $2 \%$ larger than that with the closed thumb. Although this suggests a geometric explanation for the larger drag force, the results of Fig. 3 suggest that the heuristic value of projected area is limited. Whatever the observed change of the wake in Fig. 5 is, its influence on the drag appears to be small.

The effect of thumb abduction on the lift coefficient $C_{\mathrm{L}}$ is shown in Fig. 6b. The overall shape of the dimensionless lift curve $C_{\mathrm{L}}(\varphi)=F_{\mathrm{L}} / \frac{1}{2} \rho V^{2} A$, with $L$ the lift force, is determined by both the arm and the hand. At $\varphi=0$ the hand is perpendicular to the flow, but the elliptical cross section of the arm is at $\approx 45^{\circ}$. Consequently, the lift force 
Fig. 6 (Color online) a)Drag of models with closed (adducted) and open (abducted) thumb, and fingers curved as in the neutral hand (hand 2 in Fig. 3). The yaw angles $\varphi$ were scanned up from $-20^{\circ}$ to $60^{\circ}$, and down again. $\mathbf{b}$ Same as $\mathbf{a}$ but now for the lift coefficient $C_{\mathrm{L}}$
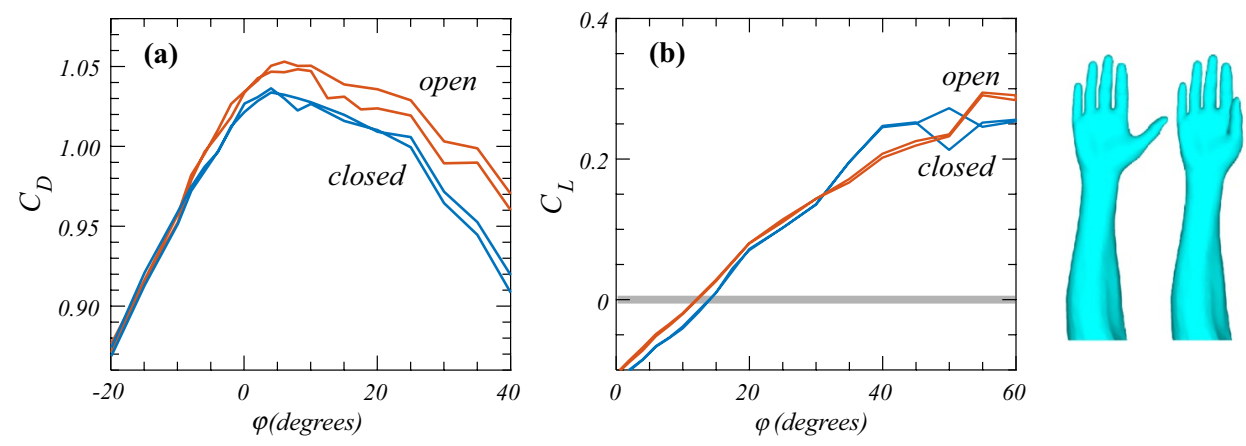

only vanishes at a nonzero value $\varphi \approx 12^{\circ}$. However, what matters is the change of the $C_{\mathrm{L}}(\varphi)$ curve with thumb abduction. The angular scan of $C_{\mathrm{L}}$ for the adducted (closed) thumb shows hysteresis. Otherwise the $C_{\mathrm{L}}$ of the abducted thumb is $\approx 25 \%$ larger, but only at large positive yaw angles with the thumb leading into the oncoming flow. Depending on the swimming style, lift can contribute significantly to propulsion (Schleihauf 1979; Cohen et al. 2015).

Concluding we find small but significant differences of the drag and lift depending on the thumb position. The differences found disagree with those in the literature. We believe that these subtle differences are inconsequential for the swimming practice.

\section{Three-dimensional flow structures}

The flow around the human hand is complex, and the question is what flow structures determine lift and drag. Clearly, the flow depends on the thumb position, and it is interesting to visualize these flow structures. In addition, we will estimate the pressure distribution on the hand using the measured 3D flow field.

We scan the flow field around the hand employing the recently developed coaxial volumetric velocimeter (CVV) by Schneiders et al. (2018). This device provides the full three-dimensional velocity field in a conical volume of $0.4 \mathrm{~m}$ height, $0.38 \times 0.43 \mathrm{~m}^{2}$ at its base and $0.13 \times 0.17 \mathrm{~m}^{2}$ at its top. This volume is moved around the arm model using a robot, and the flow field information of 15 cones is combined together (Jux et al. 2018). The locations of these cones are chosen such that there is sufficient overlap at the sides of the cones and the full area of interest around the hand is captured. Regions such as the space between the thumb and individual fingers that would otherwise be difficult to see with conventional tomographic PIV systems can be captured by a judicious choice of the cones. In order to measure the flow between the fingers, the RVV (robotic volumetric velocimetry) system looks down from the top of the hand. For the lower part of the arm, the images are acquired from the side.
For these experiments we used the Open Jet Facility of TUDelft (Lignarolo et al. 2014). The air flow is seeded with neutrally buoyant helium-filled soap bubbles (diameter $\approx 0.4 \mathrm{~mm}$ ) generated by a rake that is placed in the settling chamber of the wind tunnel. The velocity field is constructed from tracked soap bubbles. The amount of available bubbles is determined by the rate at which they are generated; this results in $\approx 700$ bubbles which are tracked over multiple time steps.

The particle tracks provide velocity information at scattered locations throughout the domain. This Lagrangian description is then averaged in space and time within cubic bins width edge size dependent on the statistical convergence of the particle velocities. Clearly, there is a trade-off between statistical accuracy and size of the bins, which in turn determines the spatial resolution.

An overview of mean-flow streamlines around the armhand model is shown in Fig. 7. Several observations can be made. First, the flow around the hand interacts with that around the forearm, so that drag studies should involve more than a hand. Second, there is a large recirculation zone and vortical motion behind the outstretched fingers. These vortices are oriented horizontally. Third, there is a lift-off of streamlines over the top of the model; this is consistent with the vertical force that was reported in Sects. 3.1 and 3.2. Finally, there is a complex low-speed flow structure around the base of the model where it meets the wind tunnel ground plate. This structure influences drag, but we assume that it does not depend on finger curvature or thumb abduction.

It is now well established that a slight spread of the fingers in front crawl swimming enhances drag and thus propulsion efficiency (Sidelnik and Young 2006; Minetti et al. 2009; Marinho et al. 2010; Lorente et al. 2012; van Houwelingen et al. 2017). Spreading the fingers does not increase the projected area of the hand, but small gaps between fingers still offer resistance to the flow. This argument leads to a prediction of an optimal finger spread.

The optimum can be derived from simple scaling arguments that are reminiscent of the Betz' prediction for the optimum power efficiency of horizontal axis wind turbines (Betz 1966). A key ingredient of this argument for the drag 


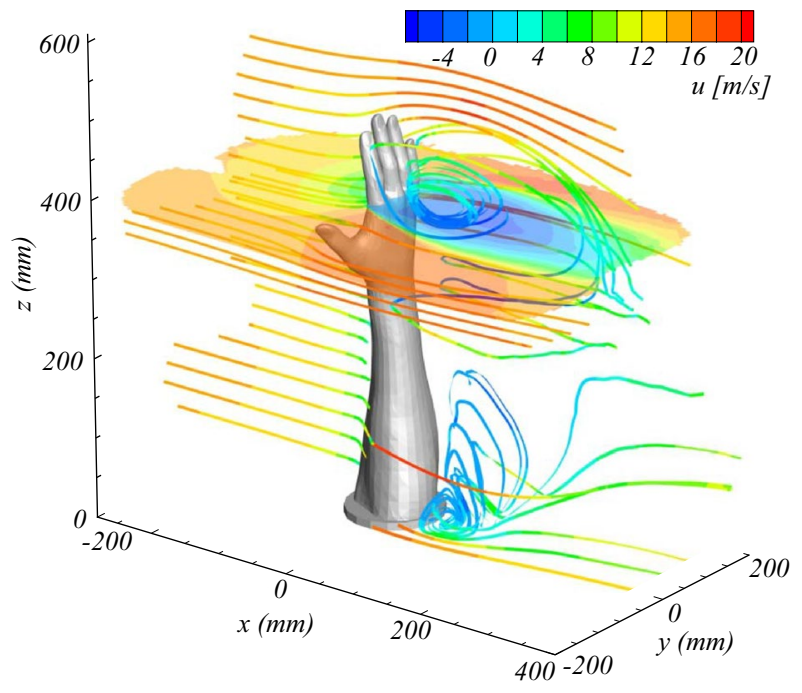

Fig. 7 (Color online) Streamlines colored by the value of the streamwise velocity illustrate the recirculating structure in the wake of the arm-hand model. The contours of $u$ are at $z=362 \mathrm{~mm}$, which is $144 \mathrm{~mm}$ below the tip of the middle finger

of the hand with spread fingers is the assumption that the flow velocity $u$ between fingers is driven by the pressure difference $\Delta p \propto \rho V^{2}$ over the hand,

$u^{2}=\xi \frac{d}{D} V^{2}$,

where $d$ is the gap size between two fingers, $D$ the diameter of a finger (see Fig. 8), $V$ the oncoming velocity, and $\xi$ is a constant of order one. Additional assumptions concern the shape of the stream tube.

Several, at first sight equally plausible variants of the Betz-type scaling arguments for the drag coefficient are possible. The one in van Houwelingen et al. (2017) used Bernoulli's theorem and predicted a drag optimum at $d / D \approx 0.4$, using $\xi \approx 10$. The one in Westerweel et al. (2016) was based on integral momentum arguments and also predicts a drag optimum at $d / D \approx 0.4$, but required a much smaller $\xi \approx 1$.

Using the RVV system, it is possible to map the flow profile between two fingers. From Fig. 8 we find that $u^{2} / V^{2} \approx 0.08$, whereas $d / D=0.4$, but the gap size decreases at smaller $z$ (closer to the hand palm). This implies $\xi \gtrsim 0.2$. Clearly, the result of the flow measurement prefers the model in Westerweel et al. (2016), which illustrates that simple scaling arguments should be selected judiciously.

\section{Pressure distribution}

Flow structures that change with the thumb position alter the pressure distribution on the hand and thus affect drag forces. As we have near-complete information on the mean
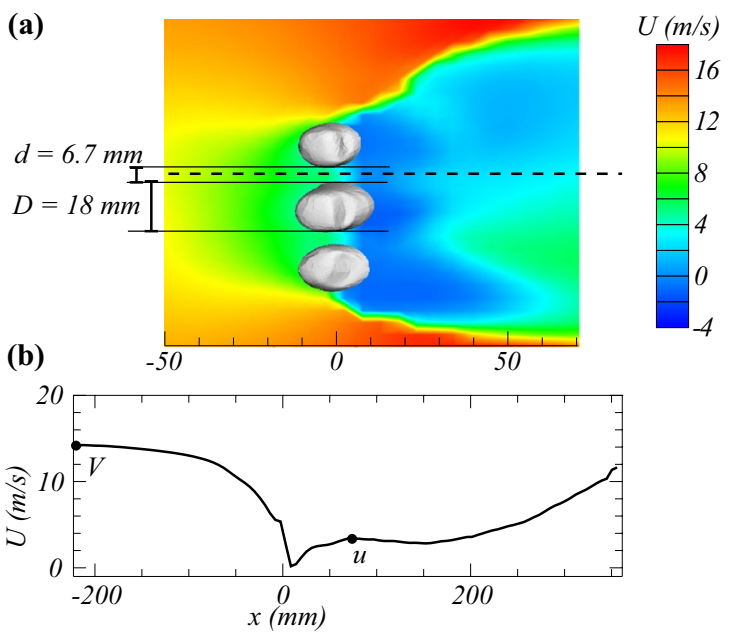

Fig. 8 (Color online) a Profile of the $U=\langle u\rangle$-component of the mean velocity between two fingers. The horizontal line along which the flow is measured is shown dashed. It is $36 \mathrm{~mm}$ below the tip of the middle finger and passes between ring and middle finger. The velocity is measured using a bin size of $10 \mathrm{~mm}$, so that the sharp drop of the velocity between the fingers is only partly resolved. b Dependence of the measured mean velocity $U$ on $x$. The dots indicate the oncoming and interdigit velocities $V, u$, respectively, that are used in Eq. (1). The gap size $d$ and finger width $D$ in Eq. (1) are indicated in a

three-dimensional flow field and Reynolds stresses, it is possible to estimate the distribution of the mean pressure on the hand and compare it to measured pressures.

Briefly, for the computation of the pressure from the measured flow field the flow domain is separated into an irrotational and a rotational part. While Bernoulli's equation provides the pressure in the first part, integration of the averaged Navier-Stokes equation gives the pressure distribution in the rotational part of the flow field. This integration starts from the boundary between the two parts and proceeds using a marching scheme (Jux et al. 2020), up to the discretized surface of the hand.

The computed pressure distribution on the hand is compared to the directly measured pressures at 16 taps spread over the hand and fingers. On the hand, the computed and measured pressures agree to within $10 \%$. The agreement of the reconstructed pressure distribution with that directly measured may be compared favorably to that of Jux et al. (2020) for the surface pressure on a sphere using the same robotic PIV technique. However, while pressure reconstruction provides valuable qualitative information, its accuracy does not yet suffice for a reproduction of the drag difference between the two thumb positions. Indeed, integration of the pressure over the hand surface leads to prediction of a $2.5 \%$ larger drag of the hand with closed thumb, opposite to what was found in the wind tunnel. From Fig. 9 it appears that abducting the thumb leads to a lower pressure at the base 
Fig. 9 (Color online) a, b)Pressure distribution on the palm side of hands with adducted (a) and abducted (b) thumb. It was computed from the measured 3D flow field and Reynolds stresses. Frames $\mathbf{c}, \mathbf{d}$ are a comparison between the pressures from PIV (open circles) and those measured with a pressure sensor (closed dots). The pressure taps on the palm side of the hand are indicated in $\mathbf{a}, \mathbf{b}$. Those at the back of the hand are approximately at the same $z$-location. The pressure at the palm center is taken as reference stagnation pressure

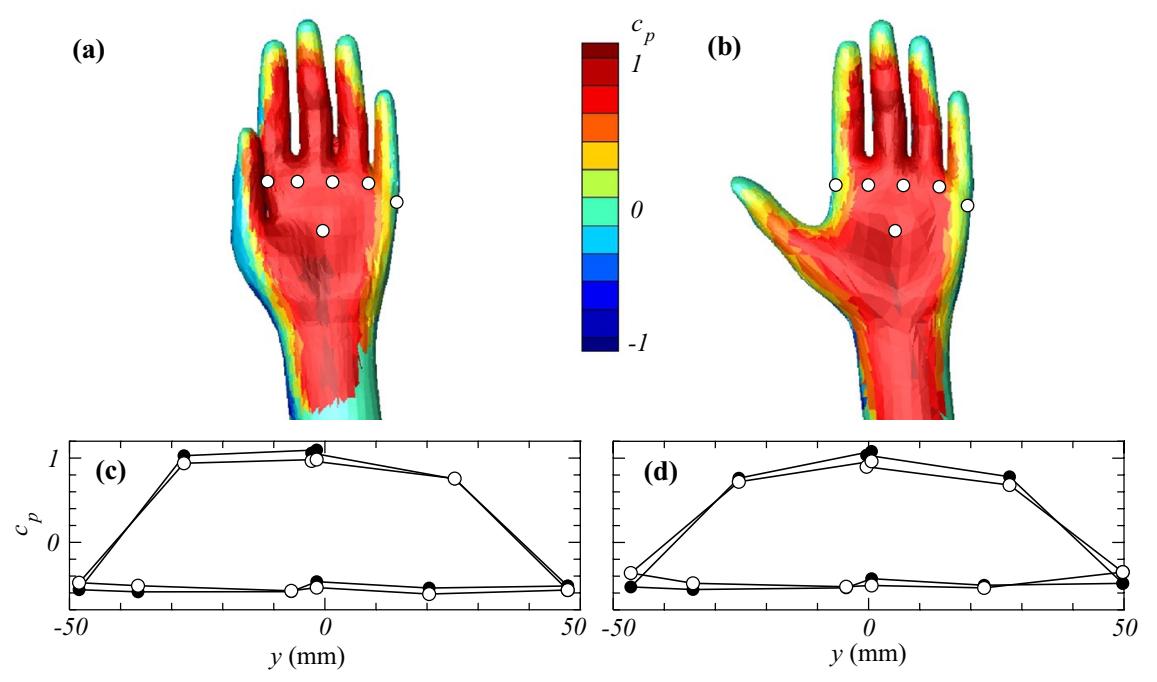

of the index finger and a lower pressure in the center of the palm. It must be compensated by a larger pressure difference across the now outstretched thumb.

These results indicate that experimental information, both about the full 3D flow field, and about the pressure about such a complex object is now accessible. However, these results also illustrate that the relation between drag and flexing of the thumb is subtle.

\section{Conclusion}

The effects of thumb and finger position on drag are small, but a small advantage in propulsion efficiency can be decisive in competitive swimming, where finishing times differ by less than a percent. Our central results are on hand curvature and thumb position, but a model for the beneficial effect of finger spreading was addressed using the 3D flow map of this study.

We find that cupping the hand does not help and that thumb abduction has a slight but significant positive effect on drag. The influence on lift is only felt at large pitch angles.

Swimming is dynamic, and a careful measurement of drag in a stationary flow is only a first step in optimizing stroke efficiency. The next step is the measurement of forces in an accelerating flow. The challenge then is to devise standard motion protocols. Also, due to the interaction with the free surface, force measurements will be less precise. Nevertheless, it was in dynamic measurements that Sidelnik and Young (2006) found a beneficial effect of a small finger spread on stroke force.

Many results presented here are at variance with those of other studies. However, in the present work all models were manufactured in the same way and were subjected to the same rigorous regime of wind tunnel drag and lift measurements that is also used for precise airfoil characterization in the used test facility.

Acknowledgements We thank Stefan Bernardy for invaluable instruction on how to operate the windtunnel and make precise measurements of aerodynamic forces, and Andrea Rubino and Jasper Ruijgrok for technical assistance.

\section{Declarations}

Conflict of interest The authors declare that there is no conflict of interest regarding the content of this article.

Open Access This article is licensed under a Creative Commons Attribution 4.0 International License, which permits use, sharing, adaptation, distribution and reproduction in any medium or format, as long as you give appropriate credit to the original author(s) and the source, provide a link to the Creative Commons licence, and indicate if changes were made. The images or other third party material in this article are included in the article's Creative Commons licence, unless indicated otherwise in a credit line to the material. If material is not included in the article's Creative Commons licence and your intended use is not permitted by statutory regulation or exceeds the permitted use, you will need to obtain permission directly from the copyright holder. To view a copy of this licence, visit http://creativecommons.org/licenses/by/4.0/.

\section{References}

Betz A (1966) Introduction to the theory of flow machines. Pergamon Press, Oxford

Bilinauskaite M, Mantha V, Rouboa A, Ziliukas P, Silva A (2013) Computational fluid dynamics study of swimmer's hand velocity, orientation and shape: contributions to hydrodynamics. BioMed Research International

Cohen R, Cleary P, Mason B, Pease D (2015) The role of the hand during freestyle swimming. J Biomech Eng 137(11):111007

Hoerner SF (1965) Fluid-dynamic drag, 2nd edn. Hoerner Fluid Dynamics

Jux C, Sciacchitano A, Schneiders JFG, Scarano F (2018) Robotic volumetric piv of a full-scale cyclist. Exp Fluids 59:74 
Jux C, Sciacchitano A, Scarano F (2020) Flow pressure evaluation on generic surfaces by robotic volumetric ptv. Meas Sci Technol 6:66

Lignarolo L, Ragni D, Krishnaswami C, Chen Q, Ferreira CS, van Bussel G (2014) Experimental analysis of the wake of a horizontalaxis wind-turbine model. Renew Energy 70:31-46

Lorente S, Cetkin E, Bello-Ochende T, Meyer J, Bejan A (2012) The constructal-law physics of why swimmers must spread their fingers and toes. J Theor Biol 308:141-146

MakeHuman-Team (2001-2016) Makehuman. https://www.makeh umancommunity.org

Marinho D, Rouboa A, Alves F, Vilas-Boas J, Machado L, Reis V, Silva A (2009) Hydrodynamic analysis of different thumb positions in swimming. J Sports Sci Med 8(1):58-66

Marinho D, Barbosa T, Reis V, Kjendlie P, Alves F, Vilas-Boas J, Machado L, Silva A, Rouboa A (2010) Swimming propulsion forces are enhanced by a small finger spread. J Appl Biomech 26(1):87-92

Minetti A, Machtsiras G, Masters J (2009) The optimum finger spacing in human swimming. J Biomech 42(13):2188-2190

Nakamura Y, Tomonari Y (1982) The effects of surface roughness on the flow past circular cylinders at high Reynolds numbers. J Fluid Mech 123:363-378

Schleihauf R (1979) Hydrodynamic analysis of swimming. In: Terauds J (ed) Swimming III: proceedings of the third international symposium of biomechanics in swimming, University of Alberta, Edmonton, Canada, International Series on Sports Sciences, vol 8, pp 70-109
Schneiders JFG, Scarano F, Jux C, Sciacchitano A (2018) Coaxial volumetric velocimetry. Meas Sci Technol 29(065):201

Shapeways (2018). https://www.shapeways.com/

Sidelnik N, Young B (2006) Optimising the freestyle swimming stroke: the effect of finger spread. Sports Eng 9(3):129-135

Takagi H, Shimizu Y, Kurashima A, Sanders R (2001) Effect of thumb abduction and adduction on hydrodynamic characteristics of a model of the human hand. In: Proceedings of swim sessions of the XIX international symposium on biomechanics in sports, $p p$ $122-126$

Timmer WA, van Rooij RPJOM (2003) Summary of the Delft University wind turbine dedicated airfoils. J Solar Energy Eng 125(4):488-496

van Houwelingen J, Willemsen DHJ, Kunnen RPJ, van Heijst GF, Grift EJ, Breugem WP, Delfos R, Westerweel J, Clercx HJH, van de Water W (2017) The effect of finger spreading on drag of the hand in human swimming. J Biomech 63:67-73

Vilas-Boas JP, Ramos RJ, Fernandes RJ, Silva AJ, Rouboa AI, Machado L, Barbosa TM, Marinho DA (2015) Hydrodynamic analysis of different finger positions in swimming: a computational fluid dynamics approach. J Appl Biomech 31(1):48-55

Westerweel J, Breugem W, Grift E, Tummers M (2016) Handiger zwemmen. Nederlands Tijdschrift voor Natuurkunde 82:170-173

Publisher's Note Springer Nature remains neutral with regard to jurisdictional claims in published maps and institutional affiliations.

\section{Authors and Affiliations}

\section{Joris van den Berg ${ }^{1} \cdot$ Rens Bazuin ${ }^{2} \cdot$ Constantin Jux ${ }^{1} \oplus \cdot$ Andrea Sciacchitano $^{1} \oplus$ - Jerry Westerweel ${ }^{2} \oplus$. Willem van de Water ${ }^{2}$ (I)}

1 Department of Aerospace Engineering, Delft University of Technology, Delft, The Netherlands
2 Laboratory for Aero and Hydrodynamics, Department of Mechanical Engineering, Delft University of Technology, Delft, The Netherlands 